\title{
Applications of Disease Benchmarks and Case Presentations
}

\author{
MICHAEL W. PILL, PharmD
}

\begin{abstract}
SUMMARY: A large dataset of integrated pharmacy and medical claims, extracted from independent third-party databases, is being combined with disease benchmarking technology to facilitate analysis of inpatient, outpatient, ancillary services, and pharmaceutical utilization and costs. The Disease Benchmarks Program was developed to create opportunities for health care decision makers to evaluate the entire health care continuum in a disease-specific fashion. The Benchmarks program is valuable because of its flexibility and because it depicts what is occurring in clinical practice. It can be customized and also show regional variations in treatment. The possible applications of benchmarking applications are discussed in the case presentations of otitis externa, acute otitis media with tympanostomy tubes, and Sjögren's syndrome.
\end{abstract}

KEYWORDS: Benchmarking, Database, Disease Management, Retrospective study, Otitis, Sjögren's syndrome, Outcome assessment (health care)

J Manag Care Pharm. 2005;11(1)(suppl S-a):S12-S18

\section{Author}

MICHAEL W. PILL, PharmD, is vice president, client services, The MCM Group, Marlton, New Jersey.

AUTHOR CORRESPONDENCE: Michael W. Pill, PharmD, Vice President,

Client Services, 108-I Centre Blvd., The MCM Group, Marlton, NJ 08053.

Tel: (856) 596-6995; Fax: (856) 596-8996;

E-mail: mpill@managedcaremeasures.com

Copyright $\odot$ 2005, Academy of Managed Care Pharmacy. All rights reserved.
L arge investments have been made in health care data warehousing and data analysis over the past 10 years. Health plans, pharmacy benefit management companies, employers, and other health care entities are increasingly interested in the ability to integrate medical and pharmacy claims data to provide even more valuable information than either dataset provides by itself. Data integration has allowed health care entities to perform robust clinical analyses as well as cost-benefit investigations in areas that were previously very difficult to research because of the separate storage for medical and pharmacy claims.

Hosts of consulting organizations have emerged to analyze the data generated by managed care organizations and provide services such as outcomes and clinical research, strategy, and market analysis. Generally, these consultant companies compile outcomes solely from internal analysis of the managed care organization's internal data, and the results are provided only to the managed care organization.

In contrast, a particularly large dataset from Pharmetrics, Inc. is being combined with disease benchmarking technology by The MCM Group, a health care marketing and communications company. The dataset is an integrated health care database (linking medical and pharmacy claims) of millions of lives organized into disease states and linked to all of the services and costs that have been captured for the patients with that disease. (See www.diseasebenchmarks.com.)

\section{Introduction to the Database}

The Total Resource Utilization (TRU) Benchmarks Disease Series combines disease-model technology, an integrated outcomes

\section{TABLE 1 Total Resource Utilization (TRU) Benchmarks Key Points}

- Built through a proprietary process that utilizes proprietary data models (TRU Disease Models) to organize and stratify episode-based claims data into easily digestible and easily accessible benchmark reports.

- A process designed to enable payers, plans, and providers to

- understand the total cost of care for a disease

- observe how disease treatment options impact resource utilization

- compare internal resource utilization data with external benchmarks and thereby gauge performance

- identify areas for improvement, adjust disease treatment and disease management strategies, and check performance after changes are implemented

- More than 100 million disease episodes (billions of claims)

- Otitis externa: 200,000 episodes evaluated

- Acute otitis media/tympanostomy tube (AOM/TT): 76,000 episodes evaluated

- Sjögren's Syndrome: 10,000 episodes evaluated

"Total Resource Utilization," "TRU," "TRU Benchmarks," "TRU Disease Models," and "Disease Benchmarks" are trademarks and/or service marks owned by Managed Care Measures, LLC. 
database, and Episode Treatment Groups (ETGs) to facilitate analysis of inpatient, outpatient, ancillary services, and pharmaceutical utilization and costs. These patient-specific utilization and cost parameters are matched to the diagnosis and linked to an episode of care. As a result of the use of ETGs, all costs, including pharmaceuticals, can be measured, tied to a diagnosis, and compared with other treatment options. Three years of the most recent and available raw claims data are extracted from an independent third-party database and condensed into a usable form (see Tables 1 and 2).

The Disease Benchmarks program was developed to create opportunities for health care decision makers to evaluate the entire health care continuum in a disease-specific fashion. It is designed to help health plans manage the provision of care as well as the cost of care. The data that is presented includes all costs, units of use, and services associated with treating a specific disease and is reported by category (Inpatient, Outpatient, Emergency Department, and Pharmacy). The costs depicted in the benchmarking publications are direct charges back to the system, that is, the actual charged cost of providing that care. Additionally, a paid-to-charged ratio is provided for each disease if users are interested in looking more specifically at actual "paid" data.

With the data and applications made available through Disease Benchmarks, health care organizations can compare their own data for a specific disease against independently generated national and regional benchmarks. The benchmarking program also allows health plans to better understand the impact that various treatment options and patterns, including pharmacotherapy, have on both clinical and economic outcomes. This provides an essential platform for more effective disease management.

\section{- Pharmaceutical Partnerships}

Pharmaceutical manufacturers have partnered with The MCM Group to provide individual Disease Benchmarks to organized customers. The manufacturers have stated a commitment to developing a benchmarking program that provides a valuable resource for health care decision makers.

The MCM Group has forged several pharmaceutical partnerships to develop health economic studies involving organizations such as Aventis for diabetes; Amgen for anemia and rheumatoid arthritis; Solvay for irritable bowel syndrome and hypogonadism; AstraZeneca for hypertension, coronary artery disease, congestive heart failure, atrial fibrillation, and deep vein thrombosis; Genentech for asthma; Roche for hepatitis C; Serono for multiple sclerosis and infertility; Wyeth/Solvay for mental health benchmarks; and Daiichi Pharmaceuticals for otitis externa, acute otitis media with tympanostomy tubes (AOM/TT), and Sjögren's syndrome.

For the purposes of this review, and to give some depth to the capabilities of the disease benchmarking process, otitis externa, $\mathrm{AOM} / \mathrm{TT}$, and Sjögren's syndrome will serve as examples for case presentations. Three separate disease models were developed that condense data aggregated from more than 27 million unique

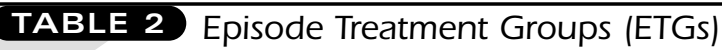

Episode treatment groups are an illness classification system matching claims to disease episodes for measuring and comparing utilization and cost of health care management. The ETG methodology accounts for case-mix adjustment, clinical homogeneity, episode building, concurrent/recurrent episodes, and shifting episodes.

ETGs are used by more than 450 health care organizations nationally to facilitate the analysis of inpatient, outpatient, ancillary, and pharmaceutical costs. Through ETGs, patient-specific costs are matched to a patient-specific diagnosis and are dynamically linked to a specific episode of care to measure resource consumption.

"ETG" or "ETGs" refers to "Episode Treatment Groups," episode-creating software owned by Symmetry Health Data Systems, Inc. "ETG", "ETGs", and "Episode Treatment Groups" are trademarks owned by Symmetry Health Data Systems, Inc. and are used under a grant of license. Episode Treatment Groups (ETGs) is protected under U.S. Patent No. 5,835,897. U.S. and foreign patents pending.

patients and 53 health plans. Each disease model identifies eligible patients using a combination of ICD-9-CM (International Classification of Diseases, Ninth Revision, Clinical Modification) diagnosis codes present on medical claims and the proprietary episode-creating software system, ETGs.

Factors believed to impact the cost of care are used to stratify and segment eligible patients into homogeneous groups for collecting and reporting data. Some of the factors include year, geography, age, gender, pharmacotherapy, comorbidities and complications, and treatment by physician specialty.

\section{Value of Disease Benchmarking}

The Benchmarks program is valuable because of its flexibility and because it depicts what is occurring in clinical practice. Of course, as in any retrospective analysis, direct clinical conclusions cannot be made. The Benchmarks program makes use of many different variables to determine which factors impact cost and utilization. It can be customized to compare different age groups, care by physician specialty, treatment by gender, or regional variations in treatment. More specific information can also be obtained, such as determination of units of use (the number of units of the actual health care deliverable used), the number of prescriptions used for a particular disease, which drug class was used, and the number of prescriptions per episode. The number of physician visits for an episode, and the number and type of specialist versus primary care physicians seen are other examples of data that can be retrieved via the disease model.

\section{Bringing It to a Practical Level}

The case presentations below show some of the practical applications of the Benchmarks program. A brief clinical background of the disease is followed by the patient selection criteria and then some of the pharmacy findings. These findings highlight the capabilities of Disease Benchmarks. For example, in the otitis externa case presentation, costs are separated by patient group and 


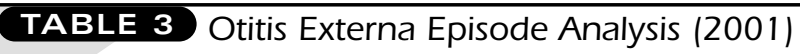

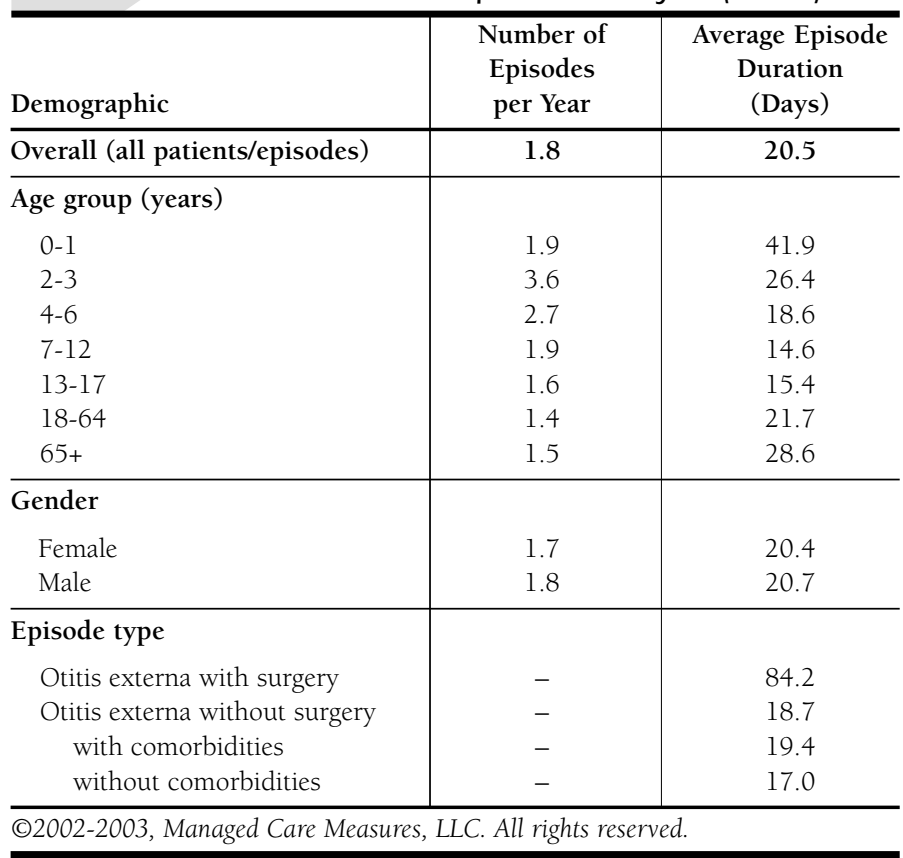

\begin{tabular}{l|c|c}
\hline TABLE 4 & $\begin{array}{c}\text { Otitis Externa Without Surgery, } \\
\text { WIth Combidities (2001) }\end{array}$ \\
\hline Inpatient & $\begin{array}{c}\text { Average Units of Use } \\
\text { per Episode }\end{array}$ & $\begin{array}{c}\text { Average Cost } \\
\text { per Episode (\$) }\end{array}$ \\
\hline Outpatient & 0.01 & 7.88 \\
\hline Emergency room & 1.86 & 126.44 \\
\hline Pharmacy & 0.17 & 19.14 \\
\hline Total & 1.47 & 56.80 \\
\hline O2002 & - & 210.26 \\
\hline
\end{tabular}

O2002-2003, Managed Care Measures, LLC. All rights reserved.

\begin{tabular}{l|c|c}
\hline TABLE 5 & $\begin{array}{c}\text { Otitis Externa Without Surgery, } \\
\text { Without Comorbidities (2001) }\end{array}$ \\
\hline & $\begin{array}{c}\text { Average Units of Use } \\
\text { per Episode }\end{array}$ & $\begin{array}{c}\text { Average Cost } \\
\text { per Episode (\$) }\end{array}$ \\
\hline Inpatient & 0.01 & 7.37 \\
\hline Outpatient & 1.66 & 123.64 \\
\hline Emergency room & 0.19 & 24.60 \\
\hline Pharmacy & 1.47 & 55.58 \\
\hline Total & - & 211.19 \\
\hline
\end{tabular}

@2002-2003, Managed Care Measures, LLC. All rights reserved reported by class of antibiotic. The AOM/TT case presentation demonstrates that patients are selected by the presence of diagnosis codes and CPT-4 (common procedural terminology) codes and are then followed over time for the occurrence of subsequent pharmacy or medical claims. The Sjögren's syndrome case presentation provides information about the demographics of the patient population and 3-year total cost trends for each medication class. Each of these measures is available for all of the disease states, but these particular disease states provide excellent material for showcasing the potential of any well-designed benchmarking-type study.

\section{Case Presentations}

\section{Case Study 1: Otitis Externa Clinical Background}

Otitis externa, ${ }^{1}$ which is characterized primarily by inflammation of the external auditory canal, affects approximately 1 in 250 people in the United States each year. ${ }^{2}$ Otitis externa is typically a bacterial and localized process that generally responds to treatment with topical antibiotic agents, but if it is not managed appropriately, it can invade the surrounding tissues and become a serious life-threatening condition..$^{3,4}$ In many cases, the associated otalgia is significant enough to disrupt daily functioning. The most common pathogen in otitis externa is Pseudomonas aeruginosa.

\section{Patient Selection}

Patients were selected for observation based on the presence of the following ETG-defined episodes of care:

- ETG 327 (otitis with major surgery)

- ETG 328 (otitis with minor surgery)

- ETG 329 (otitis without surgery)

- ETG 336 (other ENT [ear, nose, and throat] infection, with surgery)

- ETG 337 (other ENT infection, without surgery)

Of these episodes, only those containing diagnosis codes for otitis externa (ICD-9-CM codes 380.1 or 380.2) were selected for analysis. This dataset included claims from calendar years 1999-2001.

\section{Findings}

Overall, most otitis externa episodes observed were 20.5 days in duration (Table 3). The costs and units of use by episode type are analyzed into subtypes respective of the ETG groups for this disease. In otitis externa episodes without surgery, the presence of comorbidities had little impact on episode economics (Tables 4 and 5). Table 6 demonstrates the cost breakdown for otitis externa episodes without surgery by oral and otic antibiotic use.

\section{Case Study 2: Acute Otitis Media with Tympanostomy Tubes Clinical Background}

Otitis media ${ }^{5}$ is a term used to characterize inflammation of the middle ear without reference to etiology or pathogenesis. Otitis 


\begin{tabular}{|c|c|c|c|c|c|c|c|c|}
\hline & \multicolumn{4}{|c|}{ Oral Antibiotics } & \multicolumn{4}{|c|}{ Otic Antibiotics } \\
\hline & PCN (\$) & PCN-Combo (\$) & $\begin{array}{l}\text { Cephalo- } \\
\text { sporins (\$) }\end{array}$ & $\begin{array}{c}\text { Macrolide } \\
\text { Extended } \\
\text { Spectrum (\$) }\end{array}$ & $\begin{array}{c}\text { Amino- } \\
\text { Glycoside } \\
\text { Suspension }(\$)\end{array}$ & $\begin{array}{c}\text { Amino- } \\
\text { Glycoside } \\
\text { Solutions (\$) }\end{array}$ & $\begin{array}{c}\text { Quinolone } \\
\text { Suspension (\$) }\end{array}$ & $\begin{array}{l}\text { Quinolone } \\
\text { Solution (\$) }\end{array}$ \\
\hline Inpatient & 3.62 & 11.46 & 5.22 & 3.48 & 0.74 & 0.65 & 0.00 & 0.00 \\
\hline Outpatient & 94.21 & 128.79 & 127.81 & 111.33 & 86.06 & 84.51 & 105.27 & 103.21 \\
\hline Emergency room & 22.55 & 33.67 & 35.11 & 28.54 & 13.05 & 13.19 & 7.88 & 7.61 \\
\hline Pharmacy & 19.32 & 99.40 & 60.01 & 57.19 & 32.12 & 30.86 & 73.21 & 51.56 \\
\hline Total episode costs & 140 & 273 & 228 & 201 & 132 & 129 & 186 & 162 \\
\hline
\end{tabular}

(02002-2003 Managed Care Measures, LLC. All rights reserved. $P C N=$ penicillin; $P C N$-combo = penicillin-combination antibiotics.

TABLE 7 Total Episode Costs by Pharmacotherapy for Subsequent AOM Episodes (2001)

\begin{tabular}{l|c|c|c|c|c|c|c|c}
\hline & \multicolumn{4}{|c}{ Oral Antibiotics } & \multicolumn{3}{c}{ Otic Antibiotics } \\
\hline & PCN (\$) & PCN-Combo (\$) & $\begin{array}{c}\text { Cephalo- } \\
\text { sporins (\$) }\end{array}$ & $\begin{array}{c}\text { Macrolide } \\
\text { Extended } \\
\text { Spectrum (\$) }\end{array}$ & $\begin{array}{c}\text { Amino- } \\
\text { Glycoside } \\
\text { Suspension (\$) }\end{array}$ & $\begin{array}{c}\text { Amino- } \\
\text { Glycoside } \\
\text { Solutions (\$) }\end{array}$ & $\begin{array}{c}\text { Quinolone } \\
\text { Suspension (\$) }\end{array}$ & $\begin{array}{c}\text { Quinolone } \\
\text { Solution (\$) }\end{array}$ \\
\hline Inpatient & 4.71 & 17.07 & 13.21 & 9.90 & 0.00 & 0.00 & 0.00 & 10.53 \\
\hline Outpatient & 158.61 & 190.85 & 212.68 & 192.45 & 179.82 & 164.64 & 159.5 & 163.42 \\
\hline Emergency room & 14.55 & 15.90 & 16.94 & 18.95 & 7.75 & 5.59 & 3.34 & 4.40 \\
\hline Pharmacy & 40.66 & 95.98 & 92.88 & 70.76 & 32.61 & 32.73 & 71.51 & 49.74 \\
\hline Total episode costs & 219 & 320 & 336 & 292 & 220 & 203 & 234 & 228 \\
\hline
\end{tabular}

(O2002-2003, Managed Care Measures, LLC. All rights reserved. AOM = acute otitis media; $P C N=$ penicillin; $P C N$-combo = penicillin-combination antibiotics.

media results most often from a dysfunction of the eustachian tube, which is responsible for ventilating and equalizing air pressure in the the middle ear cavity. ${ }^{6} \mathrm{~A}$ short eustachian tube length may contribute to insufflation or reflux of the nasopharyngeal contents into the middle ear cavity. ${ }^{7.8}$ When this occurs, the mucosal lining becomes inflamed, limiting the ventilatory capacity of the eustachian tube. ${ }^{78}$ In addition, the mucosal lining begins to produce fluid, resulting in the accumulation of fluid in the middle ear cavity. ${ }^{8}$

TTs may be used to restore ventilation to the middle ear cavity and to decrease the incidence of AOM in otitis-prone children. ${ }^{8}$ However, otitis media recurrence after TT placement is characterized primarily by otorrhea, and is termed posttympanostomy tube otorrhea (PTTO). ${ }^{9}$

PTTO is primarily attributed to a bacterial infection, and ototopical medications are considered initial treatment. ${ }^{10}$ The antibiotic chosen should cover Pseudomonas aeruginosa and Staphylococcus species in addition to the usual pathogens seen in AOM (Streptococcus pneumoniae, Haemophilus influenzae, and Moraxella catarrhalis). ${ }^{10,11}$ TTs permit direct antibiotic application to the middle ear cavity, allowing for high concentrations at the site of infection. Topical therapy has been shown to cause fewer systemic adverse effects than oral therapy. ${ }^{9}$

\section{Patient Selection}

Patients were selected for analysis by the presence of ETG-defined episodes of care for AOM and the presence of TT placement services. Once unique patients were selected, the first AOM episode with TT placement services was identified by CPT-4 coding (codes 69433-69436). Moving forward from that point, all other AOM episodes were captured and analyzed and placed into the study period in which it occurred (i.e., 1999, 2000, or 2001). In all, there were 3 mutually exclusive and mutually exhaustive episode types observed in this analysis:

- AOM with Primary TT Placement. These AOM episodes are the first episodes reported in a patient history with TT placement services.

- AOM with Secondary TT Placement. These AOM episodes also contain TT placement services, and occur after an "AOM with Primary TT" episode.

- Subsequent AOM. These AOM episodes also occur after an "AOM with Primary TT" episode, but do not contain any TT placement services.

\section{Pharmacotherapy Segmentation}

Episodes were categorized by the presence of claims for specific 
TABLE 8 Total Episode Costs by Antibiotic Use and Episode Type (2001)

\begin{tabular}{l|c|c|c}
\hline & $\begin{array}{c}\text { AOM With } \\
\text { Primary } \\
\text { TT }(\$)^{*}\end{array}$ & $\begin{array}{c}\text { AOM With } \\
\text { Secondary } \\
\text { TT }(\$) \dagger\end{array}$ & $\begin{array}{c}\text { Subsequent } \\
\text { AOM }(\$) \neq\end{array}$ \\
\hline Oral and otic antibiotic use & 3,683 & 3,426 & 483 \\
\hline Oral antibiotic use only & 3,303 & 2,992 & 256 \\
\hline Otic antibiotic use only & 2,485 & 256 & 237 \\
\hline
\end{tabular}

(C)2002-2003, Managed Care Measures, LLC. All rights reserved.

* AOM with Primary TT = acute otitis media episodes that represent the first episode reported in a patient history with tympanostomy tube placement.

+ AOM with Secondary TT = acute otitis media episodes that also contain tympanostomy tube placement services and occur after an AOM with primary TT episode has ended (a second TT placement).

$\mp$ Subsequent $A O M=$ acute otitis media episodes that occur after a primary AOM tympanostomy tube, but do not contain any TT placement services.

\begin{tabular}{l|c|c|c}
\hline \multicolumn{4}{c}{ TABLE 9 Three-Year Cost Trends- } \\
Subsequent AOM Episodes* \\
\hline npatient & $\mathbf{1 9 9 9 ( \$ )}$ & $\mathbf{2 0 0 0 ( \$ )}$ & $\mathbf{2 0 0 1 ( \$ )}$ \\
\hline Outpatient & 14.61 & 11.44 & 11.86 \\
\hline Emergency room & 181.28 & 161.27 & 173.33 \\
\hline Pharmacy & 12.12 & 11.22 & 11.44 \\
\hline Total episode costs & 35.52 & 39.26 & 43.93 \\
\hline
\end{tabular}

(c)2002-2003, Managed Care Measures, LLC. All rights reserved.

* Subsequent $A O M=$ acute otitis media episodes that occur after a primary AOM tympanostomy tube but do not contain any TT placement services.

\begin{tabular}{l|c|c}
\hline \multicolumn{3}{c}{ TABLE 10 Sjögren's Demographics-Age and } \\
\multicolumn{3}{c}{ Gender by Patient Group (2000) } \\
\hline \multirow{2}{*}{ Demographic } & \multicolumn{2}{|c}{ Patient Group } \\
\cline { 2 - 3 } Avergren's Population & At-Risk Population \\
\hline Age group (years) & 52.4 & 49.0 \\
$0-17$ & \multicolumn{2}{|c}{$\%$ of Patients } \\
$18-35$ & 7.2 & 3.2 \\
$36-49$ & 30.6 & 12.2 \\
$50-64$ & 45.6 & 35.0 \\
$\geq 65$ & 15.0 & 37.0 \\
\hline Gender & & 12.5 \\
Female & 88.3 & \\
Male & 11.7 & 73.1 \\
\hline O2001-2002 & & 26.9 \\
\hline
\end{tabular}

C2001-2002, Managed Care Measures, LLC. All rights reserved. drug classes. To be classified with the use of a drug class, at least 1 claim from a specific drug class (identified by national drug code) was required to be present within the episode of care at any time during the episode. The use of the following drug classes was observed:

- Oral antibiotics

- Otic antibiotics, including

- aminoglycoside-based otic suspensions

- aminoglycoside-based otic solutions

- quinolone-based otic suspensions

- quinolone-based otic solutions

- Otic analgesics

- Otic corticosteroids (nonantibiotic)

\section{Findings}

Tables 7, 8, and 9 present some of the findings of the Disease Benchmarks Program for AOM/TT. Across all episode types and antibiotic therapy, the lowest total episode costs were consistently associated with the sole use of otic antibiotic therapy.

\section{Case Study 3: Sjögren's syndrome ${ }^{12}$ Clinical Background}

Sjögren's syndrome is a systemic autoimmune disease that is characterized by lymphocytic infiltration of exocrine glands, primarily lacrimal or salivary glands. ${ }^{13-16}$ Chronic and episodic damage to the lacrimal and salivary glands results in a gradual loss of ability to secrete tears and saliva, respectively, leading to worsening dry eyes and dry mouth. Many patients also experience debilitating fatigue and joint pain. ${ }^{17}$ The course of the disease is unpredictable, with symptoms that can flare or go into remission and range in intensity from mild to incapacitating. ${ }^{18,19}$

Although the hallmark symptoms are dry eyes and dry mouth, other exocrine glands can be affected resulting in impaired functioning of the respiratory, gastrointestinal, gynecologic, skin, and hepatic systems. In most patients, the primary syndrome runs a slow and benign course. Eight to 10 years often elapse from the initial symptoms to full-blown development of the disease. ${ }^{16,19}$ In this time, patients often present to multiple specialists, each of whom sees only a restricted part of the syndrome. ${ }^{19}$

There are no formal analyses of the health care costs for patients with Sjögren's syndrome. However, patients often spend years seeking an accurate diagnosis, partly because of the waxing and waning nature of the disease and partly because of the difficulties and lack of consensus in understanding, diagnosing, and treating Sjögren's syndrome. Because of the concomitant disorders, lack of adequate treatments, and out-of-pocket patient expenses, it is likely a costly disease for patients who suffer from it.

\section{Patient Selection}

Patients were selected based on the presence of one or more medical service claims with ICD-9 diagnosis code 710.2 (sicca syndrome, Sjögren's syndrome). Patients were separated into 1 of 2 
groups: those with a definitive diagnosis for Sjögren's syndrome (Sjögren's population) and those who were determined to be at risk for developing this disease (at-risk population). Patients were selected as part of the Sjögren's syndrome at-risk population based on the presence of one or more "risk factor" conditions (i.e., systemic lupus erythematosus, rheumatoid arthritis, etc.), but without the presence of diagnosis codes for Sjögren's syndrome.

Each episode consists of observations made over an interval of 365 days. This dataset included claims from calendar years 19992001.

\section{Findings}

There was a consistent distribution of data from the studied years, 1999 through 2001, with respect to the categories of average age, percentage of patients in each age group, and percentage of female and male patients. Here are some of the findings:

- Patients with Sjögren's syndrome were, on average, 52.4 years of age, and $88.3 \%$ of the total population was female (Table 10).

- Compared with internists and family/general practitioners, rheumatologists were more likely to prescribe cholinergicenhancing agents but less likely to prescribe antidepressants, oral antifungals, topical vaginal estrogens, and inhaled nasal steroids.

- The average yearly cost to treat Sjögren's syndrome was $\$ 1,938$, with approximately $46 \%$ of the charges generated from outpatient services and $47 \%$ from pharmacy services.

- Total costs of care were highest for the age group 36 to 49 years (Table 11).

- In the "at-risk" population, there was a significant amount of Sjögren's syndrome-related costs (especially for drugs common to Sjögren's), but without a diagnosis for Sjögren's syndrome, the average yearly cost was $\$ 1,208.85$, with pharmacy services accounting for nearly $78 \%$ of the total annual costs.

- The "at-risk" population used similar pharmacologic therapy as the Sjögren's syndrome population, and the total units of use (i.e., prescriptions) per episode were comparable between groups (Table 12).

- Only 14.9\% of Sjögren's syndrome episodes reflected use of cholinergic enhancers while $36.8 \%$ reflected use of antidepressants.

- The 3-year cost analysis shows an increasing trend in almost every area measured (Table 13)

\section{Pharmacotherapy Classes Studied}

- Selective cholinergic enhancers

- Nonselective cholinergic enhancers

- Disease modifying antirheumatic drugs (DMARDs)

- Oral corticosteroids

- Antidepressants

- Ophthalmic lubricants (prescription products only)

- Other ophthalmic products (includes antibiotic, anti-inflammatory, and antibiotic/anti-inflammatory combination products)

\begin{tabular}{|c|c|c|}
\hline Age Group (Years) & Pharmacy Costs (\$) & Total Costs $(\$)^{*}$ \\
\hline $0-17$ & 187.53 & $1,317.97$ \\
\hline $18-35$ & 534.27 & $1,626.96$ \\
\hline $36-49$ & 996.33 & $2,116.78$ \\
\hline $50-64$ & $1,015.69$ & $2,082.24$ \\
\hline$\geq 65$ & 602.61 & $1,348.29$ \\
\hline
\end{tabular}

O2001-2002, Managed Care Measures, LLC. All rights reserved.

* Total costs included costs from service categories: inpatient, outpatient, emergency room, and pharmacy

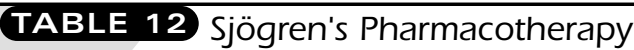
by Patient Group (2001)

\begin{tabular}{l|c|c}
\hline \multirow{2}{*}{ Drug Class } & \multicolumn{2}{|c}{ Patient Group } \\
\cline { 2 - 3 } & $\begin{array}{c}\text { Sjögren's } \\
\text { Population }\end{array}$ & $\begin{array}{c}\text { At-Risk } \\
\text { Population }\end{array}$ \\
\cline { 2 - 3 } Selective cholinergic enhancers & $\begin{array}{c}\text { \% of Episodes With Use } \\
\text { (Total Units per Episode) }\end{array}$ \\
\hline Nonselective cholinergic enhancers & $5.2(3.7)$ & $0.1(2.9)$ \\
\hline DMARDs & $9.7(4.1)$ & $0.2(3.7)$ \\
\hline Oral corticosteroids & $34.7(7.6)$ & $29.9(8.9)$ \\
\hline Antidepressants & $30.1(3.5)$ & $31.4(3.8)$ \\
\hline Ophthalmic lubricants (Rx only) & $36.8(7.3)$ & $32.4(6.9)$ \\
\hline Other ophthalmic products & $0.7(5.4)$ & $0.0(5.9)$ \\
\hline Oral antifungals & $18.6(2.1)$ & $8.1(1.8)$ \\
\hline Nasal corticosteroids & $5.1(1.7)$ & $2.1(1.7)$ \\
\hline Vaginal topical estrogens & $17.8(2.8)$ & $12.1(2.6)$ \\
\hline Vaginal topical antifungals & $5.3(2.1)$ & $2.3(1.9)$ \\
\hline O2001-2002, Managed Care & $13.0(2.2)$ & $8.1(2.0)$ \\
\hline
\end{tabular}

(O2001-2002, Managed Care Measures, LLC. All rights reserved. DMARDs = disease-modifying antirheumatic drugs.

- Oral antifungals

- Nasal corticosteroids

- Vaginal (topical) estrogens

- Vaginal (topical) antifungals

\section{How Managed Care Organizations Use the Information}

These examples help to depict actual pharmacy and medical claims activity in a way that allows decision makers to understand the demographics of the affected population, how these patients are actually being treated, and where resources are being used. This intimate look, in combination with the use of evidence-based guidelines that highlight the best available treatment options, allow a greater overall comprehensive knowledge of the disease 


\begin{tabular}{l|c|c|c}
\hline \multirow{4}{*}{$\begin{array}{c}\text { TABLE 13 Three-Year Trends of Episode Costs } \\
\text { (Sjögren's Population Only) }\end{array}$} \\
\cline { 2 - 4 } Service Category & $\mathbf{1 9 9 9 ( \$ )}$ & $\mathbf{2 0 0 0 ( \$ )}$ & $\mathbf{2 0 0 1 ( \$ )}$ \\
\hline Inpatient ancillary & 28.39 & 12.99 & 28.57 \\
\hline Inpatient facility & 39.54 & 40.32 & 95.32 \\
\hline Inpatient management & 2.03 & 2.35 & 5.17 \\
\hline Inpatient surgical & 2.00 & 1.65 & 0.09 \\
\hline Inpatient total & 71.97 & 57.31 & 129.15 \\
\hline Outpatient ancillary & 405.40 & 435.99 & 512.19 \\
\hline Outpatient management & 239.98 & 284.16 & 281.68 \\
\hline Outpatient surgical & 55.21 & 80.38 & 95.68 \\
\hline Outpatient total & 700.59 & 800.52 & 889.55 \\
\hline Emergency room & 18.57 & 20.14 & 19.05 \\
\hline Pharmacy & 501.17 & 653.18 & 901.20 \\
\hline Total episode costs & $1,292.30$ & $1,531.15$ & $1,938.95$ \\
\hline O2001-2002, Managed Care Measures, LLC. All rights reserved. \\
\hline
\end{tabular}

being treated. In this era, when decisions about pharmacy and medical coverage-and limitations of coverage-are being made and patients are directly affected, the usefulness of this knowledge cannot be understated. It helps to alert decision makers to the real versus perceived "need" of some drugs, while it may also highlight whether a decision to limit coverage is realistic for a particular patient population. Internal financial projections based on certain summaries in the benchmarking dataset may help to form clinical programs or coverage policies. Finally, managed care organizations can use the data to understand the potential impact that formulary or coverage decisions may have on the affected population.

\section{Summary}

In an environment that has a need for decreasing cost and increasing quality, benchmarking can lead to practice innovations necessary to survive. The TRU Disease Benchmarks program was developed to provide a better understanding of costs and services used in association with a particular disease. The outcomes data obtained from the benchmarking program is extremely flexible and provides insights into how the disease is treated in clinical practice, the cost associated with one episode, and which comorbidities and complications are linked with that particular disease. The real-world setting of the benchmarking database allows some assumptions to be made regarding use or misuse of a product within a defined patient population and therefore can be used as a management tool in decisions about medication coverage. It may help to answer questions such as: "How will a drug affect the pharmacy budget?" and "What might be the impact on the medical side?"

\section{DISCLOSURES}

This article is based on the proceedings of a Consultant Advisory Board meeting held on October 15, 2003, at the Academy of Managed Care Pharmacy's 2003 Educational Conference in Montreal. Quebec, Canada, and supported by an educational grant from Daiichi Pharmaceutical Corporation. Author Michael W. Pill received an honorarium from Daiichi Pharmaceutical Corporation for participation in the meeting. He discloses that he has received research grant support from Daiichi Pharmaceutical Corporation.

\section{REFERENCES}

1. Otitis Externa. Benchmarks 2002. Managed Care Measures, LLC. [Data on file, Daiichi.]

2. Holten KB, Gick J. Management of the patient with otitis externa. J Fam Pract. 2001;50:353-60.

3. Ruben RJ. Efficacy of ofloxacin and other otic preparations for otitis externa. Pediatr Infect Dis J. 2001;20:108-10.

4. Sander R. Otitis externa: a practical guide to treatment and prevention. Am Fam Phys. 2001;63:927-36.

5. Acute Otitis Media with Tympanostomy Tubes. Benchmarks 2002. Managed Care Measures, LLC. [Data on file, Daiichi.]

6. WHO/CIBA Foundation Workshop. Prevention of Hearing Impairment from Chronic Otitis Media. Report of a WHO/CIBA Foundation Workshop. 1996. Available at http://www.who.int/pbd/pdh/Docs/COMREP-8A.pdf. Accessed November 11, 2003.

7. Bluestone $\mathrm{CD}$. Acute and chronic mastoiditis and chronic suppurative otitis media. Sem Pediatr Infect Dix. 1998;9:12-26.

8. Morris MS. Tympanostomy tubes: types, indications, techniques, and complications. Otolaryngol Clin North Am. 1999;32:385-90.

9. Goldblatt EL, Dohar J, Nozza RJ, et al. Topical ofloxacin versus systemic amoxicillin/clavulanate in purulent otorrhea in children with tympanostomy tubes. Int J Pediatr Otorhinolaryngol. 1998;46:91-101.

10. Hannley MT, Denneny JC, Holzer S, for the Consensus Panel. Use of ototopical antibiotics in treating 3 common ear diseases. Otolaryngol Head Neck Surg. 2000;122:934-40.

11. Dohar JE. Old and new ototopical agents for the acute and chronic draining ear. Semin Otitis Media Manage. 1998;1:1-14.

12. Sjögren's Syndrome. Benchmarks 2001. Managed Care Measures, LLC. [Data on file, Daiichi.]

13. Al-Hashimi I. The management of Sjögren's syndrome in dental practice. JADA. 2001;132:1409-17.

14. Fox R, Stern M. Sjögren's syndrome: mechanisms of pathogenesis involve interaction of immune and neurosecretory systems. Scand J Rheumatol. 2002;31(suppl 116):3-13.

15. Fox RI, Michelson P. Approaches to the treatment of Sjögren's syndrome. J Rheumatol. 2000;27(suppl 27):15-21.

16. Moutsopoulos HM. Sjögren's Syndrome. In: Braunwald E, Hauser SL, Fauci AS, Longo DL, Kasper DL, Jameson JL, eds. Harrison's Principles of Internal Medicine. 15th ed. New York, N.Y.: McGraw-Hill; 2001:1947-49.

17. Bowman SJ. Collaborative research into outcome measures in Sjögren's syndrome. Scand J Rheumatol. 2002;31(suppl 116):23-27.

18. Sjögren's Syndrome Foundation. Available at http://www.sjogrens.com. Accessed October 23, 2003.

19. Fox R. Sjögren's Syndrome: New approaches to treatment. Available at http://www.medscape.com/viewarticle/412973. Accessed October 23, 2003. 